\title{
Bright and Dark Solitons on the Surface of Finite-Depth Fluid Below the Modulation Instability Threshold
}

\author{
I. S. Gandzha ${ }^{1}$ and Yu. V. Sedletsky ${ }^{1}$ \\ ${ }^{1}$ Institute of Physics, Nat. Acad. of Sci. of Ukraine, Prosp. Nauky 46, Kyiv 03028, Ukrain€
}

(Dated: June 22, 2018)

\begin{abstract}
We use the high-order nonlinear Schrödinger equation (NLSE) derived to model the evolution of slowly modulated wave trains with narrow spectrum on the surface of ideal finite-depth fluid. This equation is the finite-depth counterpart of celebrated Dysthe's equation, which is usually used for the same purpose in the case of infinite depth. We demonstrate that this generalized equation admits bright soliton solutions for depths below the modulation instability threshold $k h \approx 1.363$ ( $k$ being the carrier wave number and $h$ the undisturbed fluid depth), which is not possible in the case of standard NLSE. These bright solitons can exist along with the dark solitons that have recently been observed in a water wave tank [Phys. Rev. Lett. 110, 124101 (2013)].

PACS numbers: 05.45.Yv, 47.35.Bb, 92.40.Qk
\end{abstract}

Bright and dark solitons are the fundamental selflocalized modes of the optical field in nonlinear dispersive media such as waveguides, optical fibers, and photonic crystals [1]. Bright solitons are characterized by a localized intensity peak on a homogeneous background, while dark solitons can be described by a localized intensity hole on a continuous wave background [2]. Solitons are known to exist due to the balance of dispersion and nonlinearity and propagate without changing their shape and keeping their energy [3]. Bright solitons are formed when the group-velocity dispersion in an optical fiber is anomalous (or, similarly, when the nonlinearity of a planar waveguide is self-focusing). In this case, the uniform carrier wave is unstable with respect to long-wave modulations allowing for the formation of solitons. This type of instability is known as the modulation instability [4]. On the contrary, dark solitons are formed in the case of normal group-velocity dispersion in fibers (or a selfdefocusing nonlinearity in waveguides), when a uniform carrier wave is modulationally stable.

In the case of water waves, bright solitons are known to appear in the form of surface envelopes of modulated wave trains when the uniform carrier wave is modulationally unstable [5]. This happens for water depths $h$ above the modulation instability threshold, namely, at $k h>1.363, k$ being the carrier wave number. In addition to theoretical predictions, envelope solitons were observed experimentally in Refs. [6 9], mostly in the case of deep water $(k h \gg 1)$. Dark solitons can appear on shallow water below the modulation instability threshold, at $k h<1.363[10]$. They have recently been observed in a series of experiments performed in a water wave tank [11, 12].

In mathematical terms, bright and dark solitons are described by the nonlinear Schrödinger equation (NLSE) of the focusing and defocusing types, respectively [2, 5, 13]. NLSE takes into account the second-order dispersion and the phase self-modulation (cubic nonlinear term). In the general context of weakly nonlinear dispersive waves, this equation was first discussed by Benney and Newell [14]. In the case of gravity waves propagating on the surface of infinite-depth irrotational, inviscid, and incompressible fluid, NLSE was first derived by Zakharov [15]. The finite-depth NLSE was first derived by Hasimoto and Ono 16].

To achieve a better comparison with experiment and to trace nonlinear effects in the vicinity of the modulation instability threshold $k h \approx 1.363$, where the leading nonlinear term vanishes, high-order nonlinear and nonlinear-dispersive effects should be taken into consideration. In the case of infinite depth, such a high-order NLSE (HONLSE) was first derived by Dysthe [17] and then rewritten in a more general form by Trulsen et al. 18]. It includes the high-order dispersion and cubic nonlinear dispersive terms as well as an additional nonlinear dispersive term describing the input of the wave-induced mean flow. Dysthe's equation was extensively used in numerical simulations of wave evolution and showed good agreement with experiment and simulations based on fully nonlinear Euler equations [19 24].

In the case of finite depth, HONLSE is generally coupled with additional equation for the wave-induced mean flow [25, 26]. No modeling of water wave evolution has been performed with these general equations because of their complexity. Sedletsky [27] used an additional power expansion of the induced mean flow to derive a single HONLSE for the first-harmonic envelope of surface profile. This equation is the direct counterpart of Dysthe's equation [17, 18] but for the case of finite depth. Slunyaev [28] confirmed the results obtained in Ref. [27] and extended them to the next order. Gandzha et al. 29] rewrote this equation in dimensionless form and used it to model the evolution of bright solitons on finite depths. When the HONLSE terms were taken into consideration, one-soliton solutions to NLSE were transformed into quasi-soliton solutions with slowly varying amplitude. These quasi-solitons were found to propagate with nearly constant speed and possess the unique property of 
solitons to exist over long periods of time without breaking. Their speed was found to be higher than the speed of the bright NLSE solitons taken as initial conditions in computations. This phenomenon was observed earlier both in experiment and numerical modeling in the case of deep-water limit in Refs. [20, 30].

In the present work we demonstrate that the HONLSE derived in Ref. [29] admits exact solutions in the form of bright solitons below the modulation instability threshold. Such bright solitons can be observed in the same experimental setup that was used by Chabchoub et al. [11] to observe dark solitons at $k h=1.2$.

We start from the fully nonlinear Euler equations written for the potential two-dimensional waves on the surface of irrotational, inviscid, and incompressible fluid under the influence of gravity. Waves are assumed to propagate along the horizontal $x$-axis $(-\infty<x<\infty)$, and the direction of the vertical $y$-axis is selected opposite to the gravity force. The fluid is assumed to be bounded by a solid flat bed $y=-h$ at the bottom and a free surface $y=\eta(x, t)$ at the top. The atmospheric pressure is assumed to be constant on the free surface. Then the evolution of waves and associated fluid flows is governed by the following set of equations [31]:

$$
\begin{aligned}
\Phi_{x x}+\Phi_{y y} & =0,-h \leqslant y \leqslant \eta(x, t) \\
\Phi_{t}+\frac{1}{2}\left(\Phi_{x}^{2}+\Phi_{y}^{2}\right)+g \eta & =0, y=\eta(x, t) \\
\eta_{t}-\Phi_{y}+\eta_{x} \Phi_{x} & =0, y=\eta(x, t) \\
\Phi_{y} & =0, y=-h
\end{aligned}
$$

Here, $\Phi(x, y, t)$ is the velocity potential, $g$ is acceleration due to gravity, $t$ is time, and the indices designate the partial derivatives over the corresponding variables. The linear dispersion relation for wave trains with carrier frequency $\omega$ and wave number $k$ is

$$
\omega^{2}=g k \tanh (k h) \equiv g k \sigma, \quad \sigma \equiv \tanh (k h)
$$

and the carrier group speed is

$$
V_{g}=\frac{\partial \omega}{\partial k}=\frac{\omega}{2 k \sigma}\left(\left(1-\sigma^{2}\right) k h+\sigma\right) .
$$

Considering slowly modulated wave trains with narrow spectrum around the carrier frequency and wave number, the unknown free-surface displacement and velocity potential can be looked for in the form of Fourier series with slowly variable coefficients:

$$
\begin{aligned}
\left(\begin{array}{c}
\eta(x, t) \\
\Phi(x, y, t)
\end{array}\right)= & \sum_{n=-\infty}^{\infty}\left(\begin{array}{c}
\eta_{n}(x, t) \\
\Phi_{n}(x, y, t)
\end{array}\right) \exp (\mathrm{i} n(k x-\omega t)) \\
& \eta_{-n} \equiv \eta_{n}^{*}, \quad \Phi_{-n} \equiv \Phi_{n}^{*}
\end{aligned}
$$

where ${ }^{*}$ stands for complex conjugate. In the smallamplitude approximation the unknown functions $\eta_{n}$ and $\Phi_{n}$ can be all expressed in terms of the first-harmonic amplitude $\eta_{1}$ with the use of additional multi-scale power expansions [27]. In this way, the original set of equations (1) - (41) can be reduced to one evolution equation for the complex-valued amplitude $\eta_{1}(x, t)$. Introducing dimensionless time, coordinate, and amplitude

$$
\tau=\frac{\omega}{c} t, \quad \chi=k x, \quad u=2 \sqrt{c} k \eta_{1}
$$

this evolution equation can be written in the following form [29]:

$$
\begin{aligned}
u_{\tau}=- & a_{1} u_{\chi}-\mathrm{i} a_{2} u_{\chi \chi}+\mathrm{i} a_{0,0,0}|u|^{2} u \\
& +\left(a_{3} u_{\chi \chi \chi}-a_{1,0,0} u_{\chi}|u|^{2}-a_{0,0,1} u^{2} u_{\chi}^{*}\right) .
\end{aligned}
$$

This HONLSE describes the evolution of the firstharmonic envelope of the surface profile with taking into account the third-order dispersion and cubic nonlinear dispersive terms. Here, $c$ and $a_{1}$ are the dimensionless phase and group speeds, respectively,

$$
\begin{gathered}
c=-\frac{4 \sigma^{2}}{v}>0 \\
a_{1}=\frac{c k}{\omega} V_{g}=-\frac{2 \sigma}{v}\left(\left(1-\sigma^{2}\right) k h+\sigma\right)>0 \\
v=\left(\sigma^{2}-1\right)\left(3 \sigma^{2}+1\right) k^{2} h^{2}-2 \sigma\left(\sigma^{2}-1\right) k h-\sigma^{2} .
\end{gathered}
$$

The second- and third-order dispersion coefficients are

$$
\begin{gathered}
a_{2}=\frac{1}{2}, \quad a_{3}=\frac{1}{12 \sigma v} \sum_{p=0}^{3} a_{3}^{(p)}(k h)^{p}, \\
a_{3}^{(0)}=-3 \sigma^{3}, \quad a_{3}^{(1)}=-3 \sigma^{2}\left(\sigma^{2}-1\right), \\
a_{3}^{(2)}=-3 \sigma\left(\sigma^{2}-1\right)\left(3 \sigma^{2}+1\right), \\
a_{3}^{(3)}=\left(\sigma^{2}-1\right)\left(15 \sigma^{4}-2 \sigma^{2}+3\right),
\end{gathered}
$$

and the coefficient at the cubic nonlinear term is

$$
\begin{gathered}
a_{0,0,0}=-\frac{1}{16 \sigma^{4} \nu} \sum_{p=0}^{2} a_{0,0,0}^{(p)}(k h)^{p}, \\
a_{0,0,0}^{(0)}=-\sigma^{2}\left(7 \sigma^{4}-38 \sigma^{2}-9\right), \\
a_{0,0,0}^{(1)}=2 \sigma\left(3 \sigma^{6}-23 \sigma^{4}+13 \sigma^{2}-9\right), \\
a_{0,0,0}^{(2)}=\left(\sigma^{2}-1\right)^{2}\left(9 \sigma^{4}-10 \sigma^{2}+9\right), \\
\nu=\left(\sigma^{2}-1\right)^{2} k^{2} h^{2}-2 \sigma\left(\sigma^{2}+1\right) k h+\sigma^{2} .
\end{gathered}
$$

The cubic nonlinear dispersion coefficients are

$$
\begin{gathered}
\left(\begin{array}{l}
a_{1,0,0} \\
a_{0,0,1}
\end{array}\right)=\frac{1}{32 \sigma^{5} \nu^{2}} \sum_{p=0}^{5}\left(\begin{array}{c}
a_{1,0,0}^{(p)} \\
a_{0,0,1}^{(p)}
\end{array}\right)(k h)^{p}, \\
a_{1,0,0}^{(0)}=\sigma^{5}\left(\sigma^{6}-40 \sigma^{4}+193 \sigma^{2}+54\right), \\
a_{0,0,1}^{(0)}=-\sigma^{5}\left(\sigma^{6}-7 \sigma^{4}+7 \sigma^{2}-9\right), \\
a_{1,0,0}^{(1)}=-\sigma^{4}\left(\sigma^{8}-109 \sigma^{6}+517 \sigma^{4}+217 \sigma^{2}+270\right), \\
a_{0,0,1}^{(1)}=\sigma^{4}\left(\sigma^{8}+20 \sigma^{6}-158 \sigma^{4}-28 \sigma^{2}-27\right),
\end{gathered}
$$




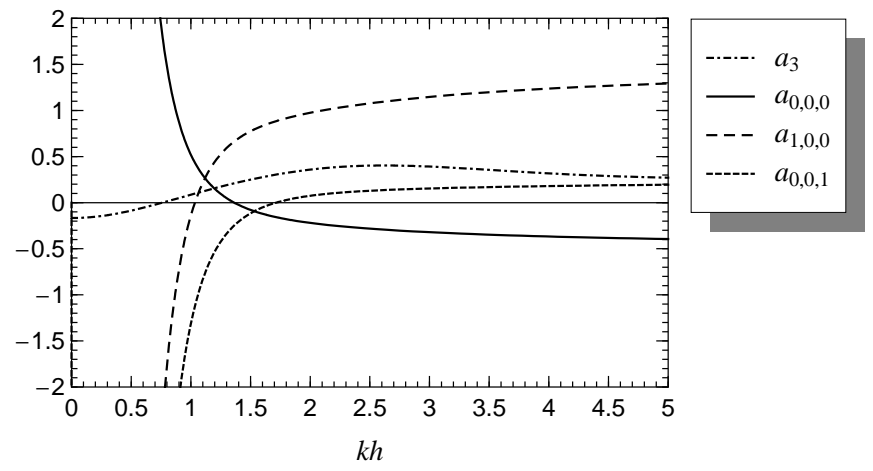

FIG. 1. HONLSE coefficients as functions of $k h$.

$$
\begin{gathered}
a_{1,0,0}^{(2)}=-2 \sigma^{3}\left(3 \sigma^{10}+18 \sigma^{8}-146 \sigma^{6}\right. \\
\left.-172 \sigma^{4}+183 \sigma^{2}-270\right), \\
a_{0,0,1}^{(2)}=2 \sigma^{3}\left(3 \sigma^{10}-63 \sigma^{8}+314 \sigma^{6}\right. \\
\left.-218 \sigma^{4}+19 \sigma^{2}+9\right), \\
a_{1,0,0}^{(3)}=2 \sigma^{2}\left(\sigma^{2}-1\right)\left(7 \sigma^{10}-58 \sigma^{8}+38 \sigma^{6}\right. \\
\left.+52 \sigma^{4}-181 \sigma^{2}+270\right), \\
a_{0,0,1}^{(3)}=-2 \sigma^{2}\left(\sigma^{2}-1\right)\left(7 \sigma^{10}-79 \sigma^{8}+282 \sigma^{6}\right. \\
\left.-154 \sigma^{4}-\sigma^{2}+9\right), \\
a_{1,0,0}^{(4)}=\sigma\left(1-\sigma^{2}\right)^{3}\left(11 \sigma^{8}-99 \sigma^{6}-61 \sigma^{4}+7 \sigma^{2}+270\right), \\
a_{0,0,1}^{(4)}=\sigma\left(\sigma^{2}-1\right)^{3}\left(11 \sigma^{8}-48 \sigma^{6}+66 \sigma^{4}+8 \sigma^{2}+27\right), \\
a_{1,0,0}^{(5)}=\left(\sigma^{2}-1\right)^{5}\left(3 \sigma^{6}-20 \sigma^{4}-21 \sigma^{2}+54\right), \\
a_{0,0,1}^{(5)}=-\left(\sigma^{2}-1\right)^{5}\left(3 \sigma^{6}+7 \sigma^{4}-11 \sigma^{2}+9\right) .
\end{gathered}
$$

The coefficients $a_{3}, a_{0,0,0}, a_{1,0,0}$, and $a_{0,0,1}$ are all real and depend only on one dimensionless parameter $k h$. Their behavior as functions of $k h$ is shown in Fig. 1 . It can be seen that Eq. (9) is valid at $k h \gtrsim 1$, where its coefficients do not diverge. At $k h \rightarrow \infty$, the following asymptotics are easily obtained:

$$
a_{3}=\frac{1}{4}, \quad a_{0,0,0}=-\frac{1}{2}, a_{1,0,0}=\frac{3}{2}, a_{0,0,1}=\frac{1}{4} .
$$

They coincide with the corresponding coefficients of Dysthe's equation 18], except for the term including the wave-induced mean flow. This term cannot be explicitly reconstructed from Eq. (9) because of the additional power expansion of the wave-induced mean flow made to derive this equation. However, it can be reconstructed from the equations generating Eq. (9), at the stage when the wave-induced mean flow has not been excluded from the equation for $u$ [27]. By setting $a_{3}=a_{1,0,0}=a_{0,0,1} \equiv 0$, Eq. (9) is reduced to the standard NLSE derived by Hasimoto and Ono 16 .

The dimensionless free surface displacement is expressed in terms of $u$ as follows

$$
k \eta=\alpha_{0}|u|^{2}+\alpha_{1} \operatorname{Re}(u \exp (\mathrm{i} \theta))+2 \alpha_{2} \operatorname{Re}\left(u^{2} \exp (2 \mathrm{i} \theta)\right),
$$

$$
\alpha_{0}=\frac{2\left(1-\sigma^{2}\right) k h+\sigma}{c \nu}, \quad \alpha_{1}=\frac{1}{\sqrt{c}}, \quad \alpha_{2}=\frac{3-\sigma^{2}}{8 c \sigma^{3}},
$$

where $\theta=k x-\omega t=\chi-c \tau$ is the wave phase. The similar expression for the velocity potential is given in Ref. [29].

The region of modulation instability of a homogeneous solution $u(\chi, \tau)=u_{0} \exp \left(i a_{0,0,0}\left|u_{0}\right|^{2} \tau\right)$ to Eq. (9) can be determined by introducing a small perturbation to the amplitude $u_{0}$ (as described, e.g., in Refs. [8, 17]):

$$
\begin{gathered}
u(\chi, \tau)=\left(u_{0}+\epsilon(\chi, \tau)\right) \exp \left(\mathbf{i} a_{0,0,0}\left|u_{0}\right|^{2} \tau\right), \\
\epsilon(\chi, \tau)=\epsilon_{0}^{+} \exp (\mathbf{i} \kappa \chi-i \Omega \tau)+\epsilon_{0}^{-} \exp \left(-\mathbf{i} \kappa \chi+i \Omega^{*} \tau\right) .
\end{gathered}
$$

Here, we assume the perturbation frequency $\Omega$ to be complex-valued and the perturbation wave number $\kappa$ to be real. Substituting this ansatz in Eq. (9) leads to the following dispersion relation between $\Omega$ and $\kappa$ :

$$
\begin{aligned}
\Omega= & \left(a_{1}+a_{1,0,0}\left|u_{0}\right|^{2}\right) \kappa+a_{3} \kappa^{3} \\
& \pm \kappa \sqrt{2 a_{2} a_{0,0,0}\left|u_{0}\right|^{2}+a_{0,0,1}^{2}\left|u_{0}\right|^{4}+a_{2}^{2} \kappa^{2}}
\end{aligned}
$$

A homogeneous solution is modulationally unstable when the perturbation exponentially grows with time. This happens when $\operatorname{Im} \Omega>0$, which effectively requires the radicand in Eq. (17) to be negative. This condition is satisfied when

$$
a_{0,0,0}<-a_{0,0,1}^{2}\left|u_{0}\right|^{2}
$$

where we took into account that $a_{2}=\frac{1}{2}$. In the NLSE case, when $a_{0,0,1} \equiv 0$, condition (18) is reduced to the well-known modulation instability criterion $a_{0,0,0}<0$, which holds true at $k h>1.363$. In the HONLSE case, this threshold slightly shifts to higher $k h$, depending on the value of $u_{0}$.

Bright and dark solitons are defined as [2]

$$
\begin{aligned}
& u_{B}(\chi, \tau)=u_{0} \operatorname{sech}(K(\chi-V \tau)) \exp (i \kappa \chi-i \Omega \tau), \\
& u_{D}(\chi, \tau)=u_{0} \tanh (K(\chi-V \tau)) \exp (i \kappa \chi-i \Omega \tau) .
\end{aligned}
$$

Here, $u_{0}, \kappa, \Omega$, and $V$, are the soliton's complex amplitude, wave number, frequency, and speed, respectively. The soliton's initial position was chosen to be located at $\chi=0$. In the NLSE case, bright one-soliton solutions of form (19) were first derived in Ref. 32]. The following relationships between the soliton parameters can be established in this case:

$$
\begin{gathered}
K=\left|u_{0}\right| \sqrt{-a_{0,0,0}} \\
\Omega=\kappa a_{1}+\frac{1}{2}\left(K^{2}-\kappa^{2}\right), V=a_{1}-\kappa .
\end{gathered}
$$

Bright NLSE solitons can exist at $a_{0,0,0}<0$, which exactly corresponds to the region where a homogeneous solution is modulationally unstable. The parameters $u_{0}$ and $\kappa$ are free parameters of the problem. However, of 


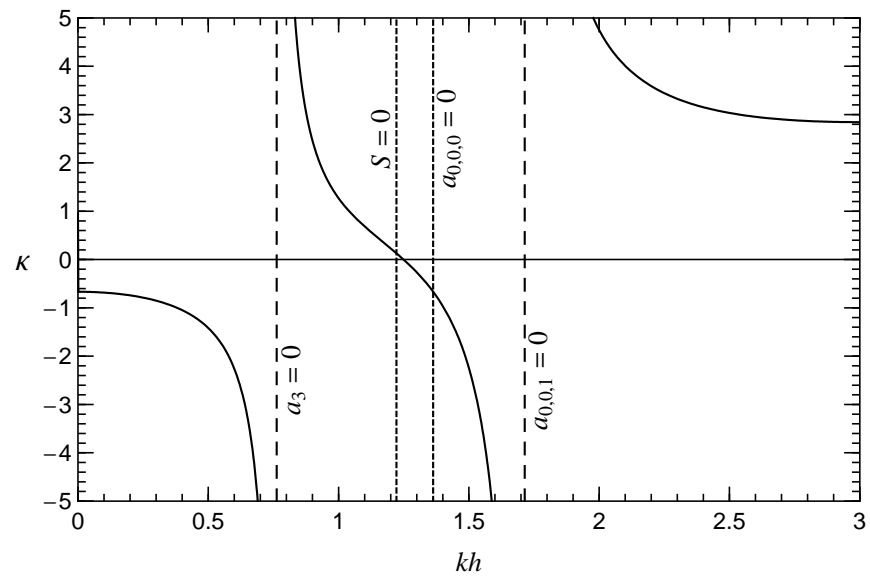

FIG. 2. Wave number $\kappa$ of the HONLSE soliton versus $k h$.

physical relevance are only such amplitudes and wave numbers that preserve the conditions of small amplitude and narrow spectrum: $\left|u_{0}\right| \ll 1, \kappa \ll 1$.

Dark solitons of form (20) are referred to as fundamental dark solitons [2]. In the NLSE context, they were first derived in a more general form in Ref. 33. Dark NLSE solitons exist below the modulation instability threshold, at $a_{0,0,0}>0$, and the corresponding relationships between the parameters are

$$
\begin{gathered}
K=\left|u_{0}\right| \sqrt{a_{0,0,0}}, \\
\Omega=\kappa a_{1}-\frac{1}{2}\left(2 K^{2}+\kappa^{2}\right), V=a_{1}-\kappa .
\end{gathered}
$$

Relations (21) and (22) are no longer valid in the HONLSE case, when the high-order terms are not set equal to zero. In this case, bright NLSE solitons are transformed into quasi-solitons, which were obtained by numerical integration of Eq. (9) in Ref. [29]. Below we prove that HONLSE (9) admits a new family of exact bright soliton solutions that exist below the modulation instability threshold $k h \approx 1.363$. Substituting ansatz (19) in Eq. (9), one can obtain the following relations:

$$
\begin{gathered}
K=\left|u_{0}\right| \sqrt{S}, \quad S=-\frac{a_{1,0,0}+a_{0,0,1}}{6 a_{3}}, \\
\kappa=\frac{a_{1,0,0}+a_{0,0,1}-6 a_{3} a_{0,0,0}}{12 a_{3} a_{0,0,1}}, \\
\Omega=\kappa a_{1}+\frac{1}{2}\left(K^{2}\left(1-6 \kappa a_{3}\right)-\kappa^{2}\left(1-2 \kappa a_{3}\right)\right), \\
V=a_{1}-\kappa+\left(3 \kappa^{2}-K^{2}\right) a_{3} .
\end{gathered}
$$

Note that such relations were derived earlier for the general form of Eq. (9) in Refs. 34 36] without referring to water waves. Anzats (19) is an exact solution to Eq. (9) when the radicand in Eq. (23a) is positive, $S>0$. This condition holds true in the following depth range:

$$
0.763 \lesssim k h \lesssim 1.222
$$

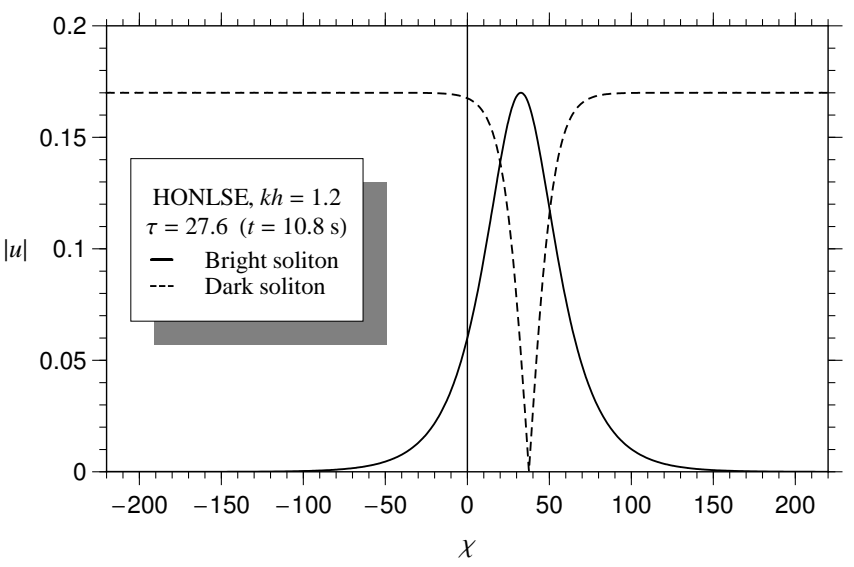

FIG. 3. Bright and dark solitons of HONLSE (9) for $k h=1.2$ and $u_{0}=0.17$. The bright soliton parameters are determined by relations (23): $K \approx 0.052, \kappa \approx 0.23, \Omega \approx 0.30, V \approx$ 1.18. The dark soliton parameters are chosen such that they closely correspond to the water-tank experiments described in Ref. [11]: $K \approx 0.066, \kappa=0, \Omega \approx-0.0043, V=a_{1} \approx 1.39$.

which lies below the modulation instability threshold. The soliton wave number $\kappa$ is not a free parameter any longer. Its behavior as a function of $k h$ is shown in Fig. 2, The condition of narrow spectrum (slow modulation) holds true only in a narrow range around $k h \approx 1.249$, where $\kappa=0$. At $k h=1.2$, we have $\kappa \approx 0.23$. This wave number can still be regarded small as compared to unity, but not so small as in the NLSE case. Figure 3 shows a bright soliton solution to HONLSE (9) along with the dark soliton that exists at the same dimensionless depth $k h=1.2$. The corresponding free surface profile computed by formula (15) is shown in Fig. (4. The parameters of the dark soliton shown in Fig. 3 were selected such that they closely correspond to the water-tank geometry and dark-soliton experiments described in Ref. [11]. These experiments were performed at the same depth $(k h=1.2)$, the carrier wave number was $k=3 \mathrm{~m}^{-1}$, and the carrier amplitude was $a=0.04 \mathrm{~m}$. The corresponding dimensionless amplitude $k a=0.12$ corresponds to the amplitude of soliton shown in Fig. 4 The evolution time $\tau=27.6(t=10.8 \mathrm{~s})$ was chosen such that the wave group travels the distance $L=V_{g} t=12.8 \mathrm{~m}$ $\left(V_{g}=1.19 \mathrm{~m} / \mathrm{s}\right)$ to the last wave gauge position along the wave tank in the experimental setup [11].

The dark soliton shown in Fig. 3 was computed by integrating HONLSE (9) numerically using the split-step Fourier technique described in [29] with the dark NLSE soliton taken as the initial wave form. On the adopted time scales, the corresponding numerical HONLSE solution identically followed the initial dark soliton shape. Deviations from NLSE can be observed on much larger time scales $(\tau \gtrsim 2000)$ in the form of slight oscillations of the wave envelope. Besides such dark quasi-solitons, HONLSE (9) admits a family of exact dark soliton solu- 


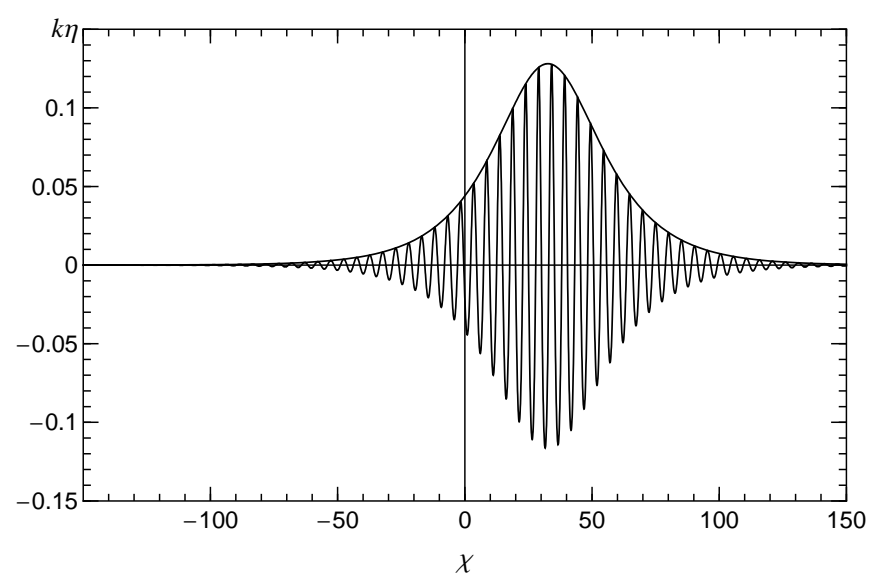

FIG. 4. Free surface profile with envelope corresponding to the bright soliton shown in Fig. $3(k h=1.2)$.

tions defined by the following set of parameters:

$$
\begin{gathered}
K=\left|u_{0}\right| \sqrt{-S}, \\
\Omega=\kappa a_{1}+K^{2}\left(6 \kappa a_{3}-1\right)-\frac{\kappa^{2}}{2}\left(1-2 \kappa a_{3}\right), \\
V=a_{1}-\kappa+\left(3 \kappa^{2}+2 K^{2}\right) a_{3},
\end{gathered}
$$

with $\kappa$ given by the same formula (23b) as for the bright solitons. These relations can be obtained in the same way as relations (23). Similar expressions for dark HONLSE solitons were also derived in Ref. [34] without referring to water waves. Dark HONLSE solitons exist at $S<0$, i.e., at $k h \gtrsim 1.222$. In contrast to bright solitons, NLSE and HONLSE dark solitons can exist in the overlapping depth ranges.

Thus, we presented for the first time new families of bright and dark solitons that exist below the modulation instability threshold for water waves on the surface of ideal finite-depth fluid. These solitons are the exact solutions to the HONLSE derived earlier in Ref. 29] to describe the evolution of slowly modulated wave trains. The results of this study can readily be used in watertank experiments to supplement the recent observations of dark solitons described in Ref. 11].

We would like to thank Dr. Denys Dutykh for helpful discussions.

gandzha@iop.kiev.ua, sedlets@iop.kiev.ua

[1] Yu.S. Kivshar and G.P. Agrawal, Optical Solitons: From Fibers to Photonic Crystals (Academic Press, San Diego, 2003).

[2] Yu. S. Kivshar and B. Luther-Davies, Phys. Rep. 298, 81 (1998).

[3] R. K. Dodd, J. C. Eilbeck, J. D. Gibbon, and H. C. Morris, Solitons and Nonlinear Wave Equations (Academic Press, London, 1984).
[4] V. E. Zakharov and L. A. Ostrovsky, Physica D 238, 540 (2009).

[5] L. Debnath, Nonlinear Water Waves (Academic Press, San Diego, 1994).

[6] H. C. Yuen and B. M. Lake, Phys. Fluids 18(8), 956 (1975).

[7] M. J. Ablowitz, J. Hammack, D. Henderson, and C. M. Schober, Phys. Rev. Lett. 84(5), 887 (2000).

[8] M. J. Ablowitz, J. Hammack, D. Henderson, and C. M. Schober, Physica D 152-153, 416 (2001).

[9] A. Slunyaev, G. F. Clauss, M. Klein, and M. Onorato, Phys. Fluids 25, 067105 (2013).

[10] D. H. Peregrine, J. Aust. Math. Soc. Series B, Appl. Math. 25, 16 (1983).

[11] A. Chabchoub, O. Kimmoun, H. Branger, N. Hoffmann, D. Proment, M. Onorato, and N. Akhmediev, Phys. Rev. Lett. 110, 124101 (2013).

[12] A. Chabchoub, O. Kimmoun, H. Branger, C. Kharif, N. Hoffmann, M. Onorato, and N. Akhmediev, Phys. Rev. E 89, 011002(R) (2014).

[13] R.H. J. Grimshaw and S. Y. Annenkov, Stud. Appl. Math. 126, 409 (2011).

[14] D. J. Benney and A. C. Newell, J. Math. Phys. 46, 133 (1967).

[15] V.E. Zakharov, J. Appl. Mech. and Tech. Phys., 9(2), 190 (1968).

[16] H. Hasimoto and H. Ono, J. Phys. Soc. Jpn. 33, 805 (1972).

[17] K. B. Dysthe, Proc. R. Soc. Lond. A 369, 105 (1979).

[18] K. Trulsen, I. Kliakhandler, K. B. Dysthe, and M. G. Velarde, Phys. Fluids 12(10), 2432 (2000).

[19] E. Lo and C. C. Mei, J. Fluid Mech. 150, 395 (1985).

[20] T. R. Akylas, J. Fluid Mech. 198, 387 (1989).

[21] D. Clamond, M. Francius, J. Grue, and C. Kharif, Eur. J. Mech. B/Fluids 25, 536 (2006).

[22] A. Slunyaev, E. Pelinovsky, A. Sergeeva, A. Chabchoub, N. Hoffmann, M. Onorato, and N. Akhmediev, Phys. Rev. E 88, 012909 (2013).

[23] A. Chabchoub, N. Hoffmann, M. Onorato, G. Genty, J. M. Dudley, and N. Akhmediev, Phys. Rev. Lett. 111, 054104 (2013).

[24] L. Shemer and L. Alperovich, Phys. Fluids 25, 051701 (2013).

[25] U. Brinch-Nielsen and I. G. Jonsson, Wave Motion 8, 455 (1986).

[26] O. Gramstad and K. Trulsen, J. Fluid Mech. 670, 404 (2011).

[27] Yu. V. Sedletsky, JETP 97, 180 (2003).

[28] A. V. Slunyaev, JETP 101, 926 (2005).

[29] I. S. Gandzha, Yu. V. Sedletsky, and D. S. Dutykh, Ukr. J. Phys. 59, 1201 (2014) arXiv:1501.05933.

[30] M.-Y. Su, Phys. Fluids 25, 2167 (1982).

[31] J. J. Stoker, Water Waves: The Mathematical Theory with Applications (John Wiley \& Sons, New York, 1992).

[32] V. E. Zakharov and A. B. Shabat, Sov. Phys. JETP 34, $62(1972)$

[33] V.E. Zakharov and A.B. Shabat, Sov. Phys. JETP 37, 823 (1973).

[34] M. J. Potasek and M. Tabor, Phys. Lett. A 154, 449 (1991).

[35] E. M. Gromov, L. V. Piskunova, and V. V. Tyutin, Phys. Lett. A 256, 153 (1999).

[36] V.I. Karpman, J. J. Rasmussen, and A. G. Shagalov, Phys. Rev. E 64, 026614 (2001). 